Supporting Information for

\title{
Diastereoselective Synthesis of 2,3,6-Trisubstituted Tetrahydropyran-4-ones via Prins Cyclizations of Enecarbamates: A Formal Synthesis of (+)-Ratjadone A
}

\author{
Kimberly N. Cossey and Raymond L. Funk* \\ Department of Chemistry, The Pennsylvania State University, \\ University Park, PA 16802
}
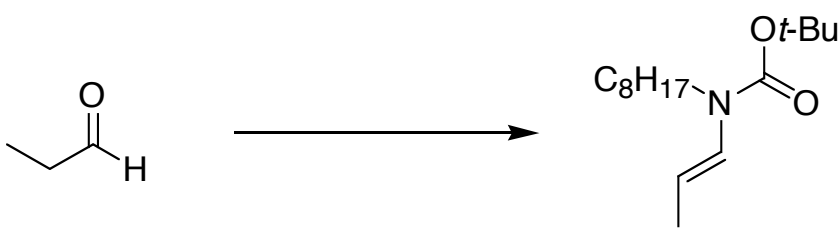

1

tert-Butyl N-octyl- $N-(E)-1$-propenylcarbamate (1). To a mixture of octylamine $(5.63 \mathrm{~g}, 43.6 \mathrm{mmol})$ and $4 \AA$ mol sieves $(2.78$ g) in $\mathrm{CH}_{2} \mathrm{Cl}_{2}(54.5 \mathrm{~mL})$ at $0{ }^{\circ} \mathrm{C}$ was added propionaldehyde $(3.46 \mathrm{~mL}$, $47.9 \mathrm{mmol}$ ) dropwise. The solution was warmed to $\mathrm{rt}$, stirred for 14 $\mathrm{h}$, and decanted. To the resultant solution at $0{ }^{\circ} \mathrm{C}$ were added $\mathrm{CH}_{2} \mathrm{Cl}_{2}$ (54.5 $\mathrm{mL})$, di-tert-butyl dicarbonate $(9.51 \mathrm{~g}, 43.6 \mathrm{mmol})$, and diisopropylethylamine $(11.4 \mathrm{~mL}, 65.4 \mathrm{mmol})$. The mixture was warmed to $\mathrm{rt}$ and stirred for $18 \mathrm{~h}$. The solution was concentrated, and toluene $(100 \mathrm{~mL})$ was added. The solution was heated at $80{ }^{\circ} \mathrm{C}$ for $36 \mathrm{~h}$ and then concentrated. Purification by silica-gel chromatography (ethyl acetate-hexanes, $1: 99)$ gave a colorless oil (8.64 g, 74\%); ${ }^{1} \mathrm{H}$ NMR (300 MHz, d d $_{6}$ acetone) $\delta 0.87$ (br t, $J=6.8 \mathrm{~Hz}$, $3 \mathrm{H}), 1.30(\mathrm{~m}, 10 \mathrm{H}), 1.46(\mathrm{br} \mathrm{s}, 9 \mathrm{H}), 1.52(\mathrm{~m}, 2 \mathrm{H}), 1.66(\mathrm{dd}, J=1.4$, $6.5 \mathrm{~Hz}, 3 \mathrm{H}$ ), 3.44 (br t, $J=7.3 \mathrm{~Hz}, 2 \mathrm{H}$ ), 4.85 (dq, $J=6.7,14.2 \mathrm{~Hz}$, $1 \mathrm{H}), 6.89(\mathrm{~m}, 1 \mathrm{H}) ;{ }^{13} \mathrm{C}$ NMR (75 MHz, d6-acetone) $\delta$ 14.3, 15.6, 23.3, 27.5, 28.0 (br), 28.4, 30.0, 32.5, 44.1 (br), 80.6, 102.5, 128.6, 153.2 (br); IR (neat) 1706, $1167 \mathrm{~cm}^{-1}$; HRMS $\left(\mathrm{M}+\mathrm{H}^{+}\right)$calcd for $\mathrm{C}_{16} \mathrm{H}_{32} \mathrm{NO}_{2}$ 270.2428 , found 270.2409 . 


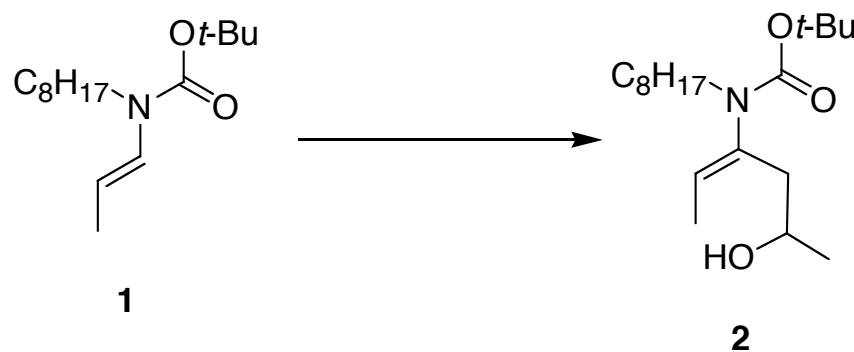

tert-Butyl $N$-octyl- $N$-[(E)-1-(2-hydroxypropyl)propenyl]-

carbamate (2). To a solution of enecarbamate 1 (160 mg, 0.59 $\mathrm{mmol})$ in THF $(0.60 \mathrm{~mL})$ at $-78^{\circ} \mathrm{C}$ was added dropwise over $4 \mathrm{~min} t$ BuLi $(1.7 \mathrm{M}$ in pentane, $326 \mu \mathrm{L}, 0.55 \mathrm{mmol})$. The solution was stirred at $-78{ }^{\circ} \mathrm{C}$ for $30 \mathrm{~min}$, warmed to $-50{ }^{\circ} \mathrm{C}$ and stirred for an additional $30 \mathrm{~min}$. The mixture was cooled to $-78{ }^{\circ} \mathrm{C}$ and $\mathrm{BF}_{3} \cdot \mathrm{OEt}_{2}$ ( $70 \mu \mathrm{L}, 0.55 \mathrm{mmol}$ ) was added, followed by propylene oxide $(3.2 \mathrm{M}$ in THF, $125 \mu \mathrm{L}, 0.39 \mathrm{mmol}$ ). The solution was stirred at $-78{ }^{\circ} \mathrm{C}$ for $20 \mathrm{~h}$. The mixture was quenched with saturated aqueous $\mathrm{NH}_{4} \mathrm{Cl}$ and extracted with ethyl acetate. The combined organic extracts were washed with $\mathrm{H}_{2} \mathrm{O}$, brine, dried $\left(\mathrm{Na}_{2} \mathrm{SO}_{4}\right)$, and concentrated. Purification by silica-gel chromatography $\left(\mathrm{CH}_{2} \mathrm{Cl}_{2}\right.$, followed by $\mathrm{Et}_{2} \mathrm{O}$ $\left.\mathrm{CH}_{2} \mathrm{Cl}_{2}, 1: 19\right)$ gave a colorless oil $(88 \mathrm{mg}, 67 \%) ;{ }^{1} \mathrm{H}$ NMR $(300 \mathrm{MHz}$, $\left.\mathrm{CDCl}_{3}\right) \delta 0.84($ br t, $J=6.7 \mathrm{~Hz}, 3 \mathrm{H}), 1.19(\mathrm{~d}, J=6.2 \mathrm{~Hz}, 3 \mathrm{H}), 1.24(\mathrm{~m}$, $10 \mathrm{H}), 1.42$ (s, 9H), $1.46(\mathrm{~m}, 2 \mathrm{H}), 1.68$ (d, $J=7.0 \mathrm{~Hz}, 3 \mathrm{H}), 2.05$ (br d, $J=14.1 \mathrm{~Hz}, 1 \mathrm{H}), 2.29(\mathrm{dd}, J=10.1,14.1 \mathrm{~Hz}, 1 \mathrm{H}), 2.96(\mathrm{dt}, J=7.0$, $14.2 \mathrm{~Hz}, 1 \mathrm{H}), 3.32(\mathrm{~m}, 1 \mathrm{H}), 3.78(\mathrm{~m}, 1 \mathrm{H}), 4.30$ (v br s, $1 \mathrm{H}), 5.51$ (br q, $J=6.9 \mathrm{~Hz}, 1 \mathrm{H}) ;{ }^{13} \mathrm{C}$ NMR $\left(75 \mathrm{MHz}, \mathrm{CDCl}_{3}\right) \delta 13.2,14.0,22.2,22.5$, 26.6, 28.0, 28.3, 28.5, 29.1, 29.2, 31.7, 38.8, 47.3, 64.4, 80.1, 128.1, 136.1, 156.6; IR (neat) 3456, 1693, $1165 \mathrm{~cm}^{-1}$; HRMS $\left(\mathrm{M}+\mathrm{H}^{+}\right)$calcd for $\mathrm{C}_{19} \mathrm{H}_{38} \mathrm{NO}_{3} 328.2846$, found 328.2820.
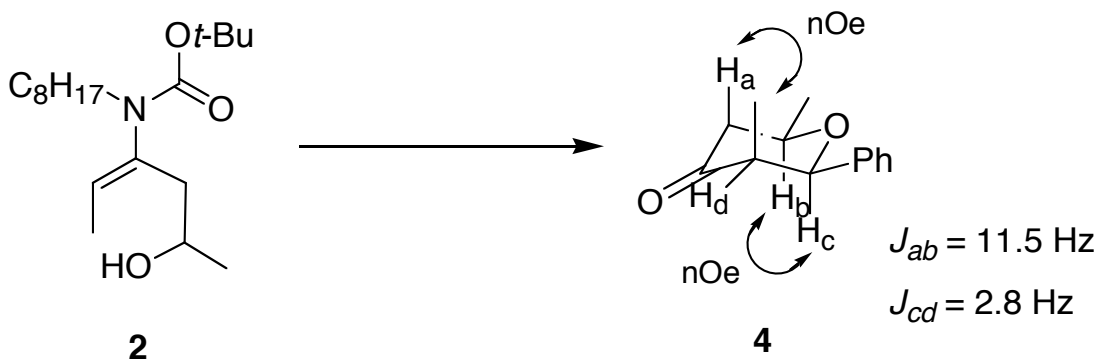

3,6-Dimethyl-2-phenyltetrahydropyran-4-one 4. To a solution of alcohol 2 (40 mg, $0.12 \mathrm{mmol})$ in $\mathrm{CH}_{2} \mathrm{Cl}_{2}(1.02 \mathrm{~mL})$ at $0{ }^{\circ} \mathrm{C}$ 
were added benzaldehyde $(14 \mu \mathrm{L}, 0.13 \mathrm{mmol})$ and $\mathrm{InCl}_{3}(14 \mathrm{mg}$, $0.06 \mathrm{mmol}$ ). The solution was stirred at $\mathrm{rt}$ for $7 \mathrm{~h}$. The mixture was quenched with saturated aqueous $\mathrm{NH}_{4} \mathrm{Cl}$ and extracted with $\mathrm{CH}_{2} \mathrm{Cl}_{2}$. The combined organic extracts were washed with $\mathrm{H}_{2} \mathrm{O}$, brine, dried $\left(\mathrm{Na}_{2} \mathrm{SO}_{4}\right)$, and concentrated. Purification by silica-gel chromatography $\left(\mathrm{Et}_{2} \mathrm{O}\right.$-hexanes, $\left.1: 4\right)$ gave a colorless oil $(23 \mathrm{mg}$, 92\%); ${ }^{1} \mathrm{H}$ NMR (400 MHz, $\mathrm{CDCl}_{3}$ ) $\delta 0.90(\mathrm{~d}, J=7.2 \mathrm{~Hz}, 3 \mathrm{H}), 1.44(\mathrm{~d}, J$ $=6.1 \mathrm{~Hz}, 3 \mathrm{H}), 2.33(\mathrm{ddd}, J=1.3,2.8,14.5 \mathrm{~Hz}, 1 \mathrm{H}), 2.54(\mathrm{dd}, J=$ $11.5,14.5 \mathrm{~Hz}, 1 \mathrm{H}), 2.62(\mathrm{qdd}, J=1.3,2.8,7.2 \mathrm{~Hz}, 1 \mathrm{H}), 3.90(\mathrm{dqd}, J=$ 2.8, 6.1, $11.5 \mathrm{~Hz}, 1 \mathrm{H}), 4.81(\mathrm{~d}, J=2.8 \mathrm{~Hz}, 1 \mathrm{H}), 7.24-7.41(\mathrm{~m}, 5 \mathrm{H})$; ${ }^{13} \mathrm{C}$ NMR $\left(50 \mathrm{MHz}, \mathrm{CDCl}_{3}\right) \delta 11.3,22.1,45.5,50.7,73.6,80.0,125.5$, 127.3, 128.2, 139.0, 211.4; IR (neat) 2980, 1711, $1107 \mathrm{~cm}^{-1}$; HRMS $\left(\mathrm{M}+\mathrm{H}^{+}\right)$calcd for $\mathrm{C}_{13} \mathrm{H}_{17} \mathrm{O}_{2}$ 205.1223, found 205.1227.

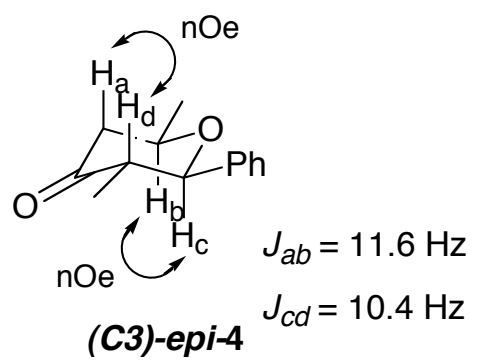

Epimerization of 3,6-Dimethyl-2-phenyltetrahydropyran-4one 4 . To a mixture of $4(16 \mathrm{mg}, 0.08 \mathrm{mmol})$ and silica gel (160 $\mathrm{mg})$ in $\mathrm{CH}_{2} \mathrm{Cl}_{2}(0.5 \mathrm{~mL})$ was added triethylamine $(500 \mu \mathrm{L}, 3.6 \mathrm{mmol})$. The mixture was stirred at $\mathrm{rt}$ for $3 \mathrm{~d}$, filtered and concentrated to give (C3)-epi-4 as a colorless oil (15 mg, 94\%); ${ }^{1} \mathrm{H}$ NMR (400 MHz, $\left.\mathrm{CDCl}_{3}\right) \delta 0.80(\mathrm{~d}, J=6.7 \mathrm{~Hz}, 3 \mathrm{H}), 1.38(\mathrm{~d}, J=6.1 \mathrm{~Hz}, 3 \mathrm{H}), 2.51(\mathrm{~m}$, 2H), $2.62(\mathrm{dq}, J=6.7,10.4 \mathrm{~Hz}, 1 \mathrm{H}), 3.92(\mathrm{ddd}, J=6.1,11.6,14.1 \mathrm{~Hz}$, $1 \mathrm{H}), 4.17(\mathrm{~d}, J=10.4 \mathrm{~Hz}, 1 \mathrm{H}), 7.27-7.43(\mathrm{~m}, 5 \mathrm{H}) ;{ }^{13} \mathrm{C}$ NMR $(75 \mathrm{MHz}$, $\left.\mathrm{CDCl}_{3}\right) \delta$ 9.4, 22.3, 49.8, 51.2, 74.2, 85.9, 124.8, 127.2, 128.4, 128.6, 139.8, 208.3. 


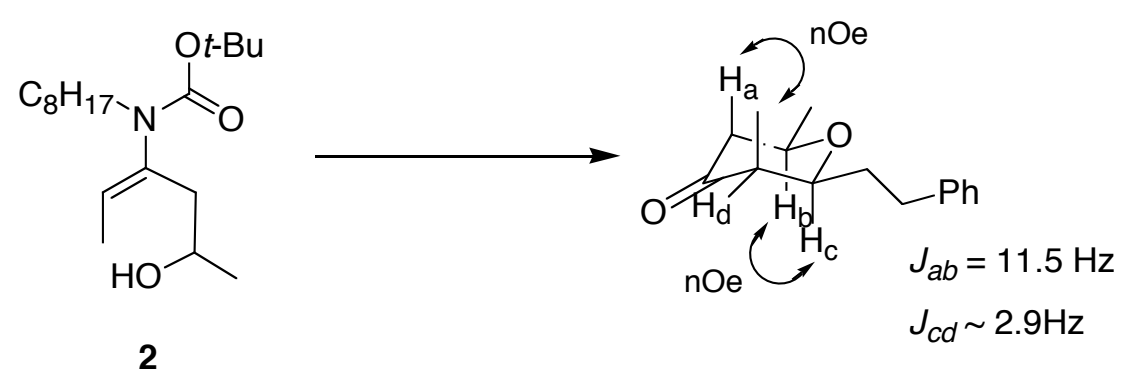

3,6-Dimethyl-2-phenethyltetrahydropyran-4-one of entry 1. To a solution of alcohol $2(131 \mathrm{mg}, 0.40 \mathrm{mmol})$ in $\mathrm{CH}_{2} \mathrm{Cl}_{2}(3.3$ $\mathrm{mL})$ at $0{ }^{\circ} \mathrm{C}$ were added hydrocinnamaldehyde $(106 \mu \mathrm{L}, 0.80 \mathrm{mmol})$ and $\mathrm{InCl}_{3} 44 \mathrm{mg}, 0.20 \mathrm{mmol}$ ). The solution was warmed to rt and stirred for $5 \mathrm{~h}$. The mixture was quenched with saturated aqueous $\mathrm{NH}_{4} \mathrm{Cl}$ and extracted with $\mathrm{CH}_{2} \mathrm{Cl}_{2}$. The combined organic extracts were washed with $\mathrm{H}_{2} \mathrm{O}$, brine, dried $\left(\mathrm{Na}_{2} \mathrm{SO}_{4}\right)$, and concentrated. Purification by silica-gel chromatography $\left(\mathrm{CH}_{2} \mathrm{Cl}_{2}\right.$-hexanes, $\left.1: 1\right)$ gave a colorless oil (75 mg, 80\%); ${ }^{1} \mathrm{H}$ NMR $\left(400 \mathrm{MHz}, \mathrm{CDCl}_{3}\right) \delta 1.16$ $(\mathrm{d}, J=7.2 \mathrm{~Hz}, 3 \mathrm{H}), 1.37(\mathrm{~d}, J=6.1 \mathrm{~Hz}, 3 \mathrm{H}), 1.66(\mathrm{~m}, 1 \mathrm{H}), 2.05(\mathrm{~m}$, $1 \mathrm{H}), 2.24(\mathrm{dt}, J=1.4,14.6 \mathrm{~Hz}, 1 \mathrm{H}), 2.33$ (qt, $J=1.2,7.2 \mathrm{~Hz}, 1 \mathrm{H})$, $2.42(\mathrm{dd}, J=11.5,14.5 \mathrm{~Hz}, 1 \mathrm{H}), 2.68$ (ddd, $J=7.5,8.6,13.9 \mathrm{~Hz}, 1 \mathrm{H})$, 2.83 (ddd, $J=5.4,9.3,13.7 \mathrm{~Hz}, 1 \mathrm{H}$ ), 3.56 (br dt, $J=3.0,8.8 \mathrm{~Hz}, 1 \mathrm{H}$ ), 3.69 (ddd, $J=2.7,6.1,11.6 \mathrm{~Hz}, 1 \mathrm{H}), 7.18-7.36(\mathrm{~m}, 5 \mathrm{H}) ;{ }^{13} \mathrm{C}$ NMR $\left(100 \mathrm{MHz}, \mathrm{CDCl}_{3}\right) \delta 10.7,22.1,32.0,33.4,45.8,48.7,73.4,77.7$, 124.7, 125.9, 128.4, 141.4, 211.5; IR (neat) 2973, 1714, $1087 \mathrm{~cm}^{-1}$; HRMS $\left(\mathrm{M}+\mathrm{H}^{+}\right)$calcd for $\mathrm{C}_{15} \mathrm{H}_{21} \mathrm{O}_{2}$ 233.1536, found 233.1540.
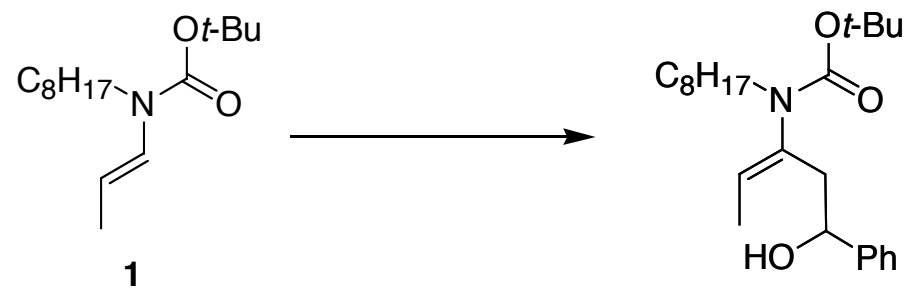

$t$ e $r t-B u t y 1$

$N$-octyl- $N$-[(E)-1-(2-hydroxy-2-phenylethyl)propenyl]carbamate. To a solution of enecarbamate $1(300 \mathrm{mg}$, $1.1 \mathrm{mmol})$ in THF $(1.1 \mathrm{~mL})$ at $-78{ }^{\circ} \mathrm{C}$ was added dropwise over $5 \mathrm{~min}$ $t$-BuLi $(1.7 \mathrm{M}$ in pentane, $786 \mu \mathrm{L}, 1.3 \mathrm{mmol})$. The solution was stirred at $-78{ }^{\circ} \mathrm{C}$ for $30 \mathrm{~min}$, warmed to $-50{ }^{\circ} \mathrm{C}$ and stirred for an additional $30 \mathrm{~min}$. The mixture was cooled to $-78{ }^{\circ} \mathrm{C}$ and $\mathrm{BF}_{3} \cdot \mathrm{OEt}_{2}$ $(141 \mu \mathrm{L}, 1.1 \mathrm{mmol})$ was added, followed by styrene oxide $(106 \mu \mathrm{L}$, $0.93 \mathrm{mmol})$. The solution was stirred at $-78^{\circ} \mathrm{C}$ for $3 \mathrm{~h}$, quenched 
with saturated aqueous $\mathrm{NH}_{4} \mathrm{Cl}$, and extracted with ethyl acetate. The combined organic extracts were washed with $\mathrm{H}_{2} \mathrm{O}$, brine, dried $\left(\mathrm{Na}_{2} \mathrm{SO}_{4}\right)$, and concentrated. Purification by silica-gel chromatography (ethyl acetate-hexanes, $1: 19$ ) gave a colorless oil (277 mg, 77\%); ${ }^{1} \mathrm{H}$ NMR (300 MHz, $\mathrm{CDCl}_{3}$ ) $\delta 0.90$ (br t, $J=6.4 \mathrm{~Hz}$, 3H), 1.30 (m, 10H), 1.53 (br s, 9H), $1.56(\mathrm{~m}, 2 \mathrm{H}), 1.77$ (d, J=6.9 Hz, 3H), 2.37 (br d, $14.2 \mathrm{~Hz}, 1 \mathrm{H}), 2.63$ (dd, $J=10.8,13.9 \mathrm{~Hz}, 1 \mathrm{H}), 3.09$ (dt, $J=7.0,14.0 \mathrm{~Hz}, 1 \mathrm{H}), 3.44$ (br m, 1H), 4.77 (br d, $J=10.5 \mathrm{~Hz}$, $1 \mathrm{H}$ ), 4.84 (v br s, 1H), 5.63 (br q, $J=6.8 \mathrm{~Hz}, 1 \mathrm{H}$ ), 7.23-7.47 (m, 5H); ${ }^{13} \mathrm{C}$ NMR $\left(75 \mathrm{MHz}, \mathrm{CDCl}_{3}\right) \delta 13.2,14.0,22.5,26.7,28.3,29.2,31.7$, $40.0,47.6,70.5,80.4,125.5,127.0,128.2,128.7,135.8,143.8$, 156.6; IR (neat) 3426, 1699, $1163 \mathrm{~cm}^{-1}$; HRMS $\left(\mathrm{M}+\mathrm{H}^{+}\right)$calcd for $\mathrm{C}_{24} \mathrm{H}_{40} \mathrm{NO}_{3}$ 390.3003, found 390.2995.

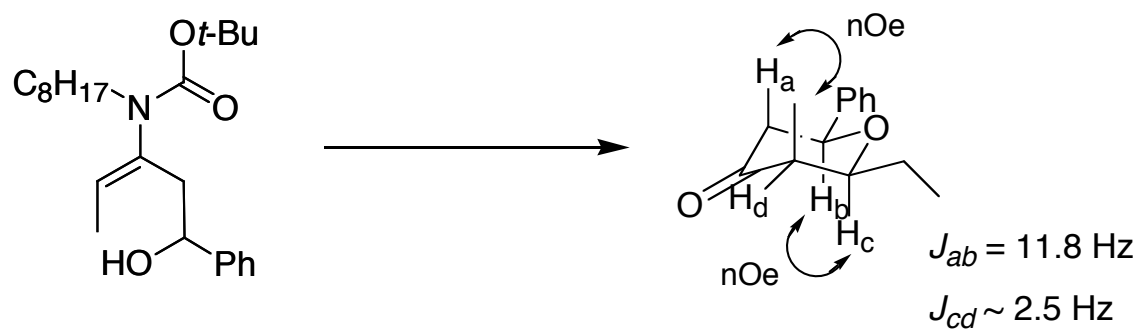

2-Ethyl-3-methyl-6-phenyltetrahydropyran-4-one of entry 2 . To a solution of tert-butyl $N$-octyl- $N-[(E)-1-(2$-hydroxy-2phenylethyl)-propenyl]carbamate (200 $\mathrm{mg}, 0.51 \mathrm{mmol}$ ) in $\mathrm{CH}_{2} \mathrm{Cl}_{2}$ $(4.3 \mathrm{~mL})$ at $0{ }^{\circ} \mathrm{C}$ were added propionaldehyde $(74 \mu \mathrm{L}, 1.03 \mathrm{mmol})$ and $\mathrm{InCl}_{3}(57 \mathrm{mg}, 0.26 \mathrm{mmol})$. The solution was stirred at $0{ }^{\circ} \mathrm{C}$ for $1 \mathrm{~h}$, warmed to rt, and stirred for $20 \mathrm{~h}$. The mixture was quenched with saturated aqueous $\mathrm{NH}_{4} \mathrm{Cl}$ and extracted with $\mathrm{CH}_{2} \mathrm{Cl}_{2}$. The combined organic extracts were washed with $\mathrm{H}_{2} \mathrm{O}$, brine, dried $\left(\mathrm{Na}_{2} \mathrm{SO}_{4}\right)$, and concentrated. Purification by silica-gel chromatography (ethyl acetate-hexanes, $1: 19$ ) gave a colorless oil (72 mg, 70\%); ${ }^{1} \mathrm{H}$ NMR (300 MHz, $\left.\mathrm{CDCl}_{3}\right) \delta 0.98(\mathrm{t}, J=7.5 \mathrm{~Hz}, 3 \mathrm{H})$, $1.21(\mathrm{~d}, J=7.2 \mathrm{~Hz}, 3 \mathrm{H}), 1.53$ (ddq, $J=6.0,7.5,15.0 \mathrm{~Hz}, 1 \mathrm{H}), 1.82$ (dq, $J=7.5,15.0 \mathrm{~Hz}, 1 \mathrm{H}), 2.44-2.53(\mathrm{~m}, 2 \mathrm{H}), 2.74(\mathrm{dd}, J=11.7,14.5$ $\mathrm{Hz}, 1 \mathrm{H}), 3.66(\mathrm{ddd}, J=2.5,5.9,7.7 \mathrm{~Hz}, 1 \mathrm{H}), 4.62(\mathrm{dd}, J=2.9,11.8$ $\mathrm{Hz}, 1 \mathrm{H}), 7.29-7.42(\mathrm{~m}, 5 \mathrm{H}) ;{ }^{13} \mathrm{C} \mathrm{NMR}\left(75 \mathrm{MHz} \mathrm{CDCl}_{3}\right) \delta 10.1,10.6$, 24.7, 45.8, 48.5, 78.8, 80.8, 125.5, 127.9, 128.5, 141.1, 211.2; IR (neat) 2970, 1714, $1097 \mathrm{~cm}^{-1}$; HRMS $\left(\mathrm{M}+\mathrm{H}^{+}\right)$calcd for $\mathrm{C}_{14} \mathrm{H}_{19} \mathrm{O}_{2}$ 219.1380, found 219.1379. 


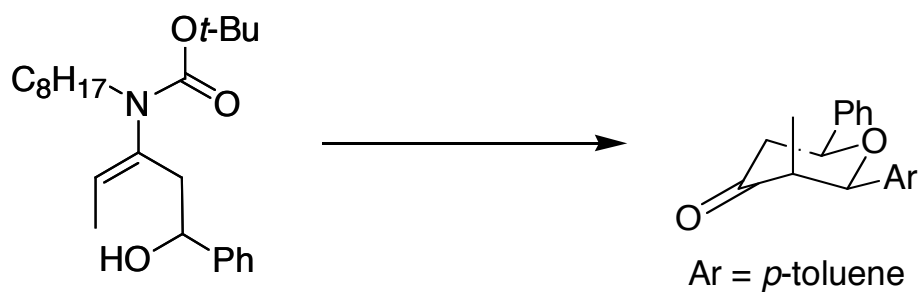

3-Methyl-6-phenyl-2-p-tolyltetrahydropyran-4-one of entry 3. To a solution of tert-butyl $N$-octyl- $N$ - $[(E)-1-(2$-hydroxy-2phenylethyl)-propenyl]carbamate $(87 \mathrm{mg}, 0.22 \mathrm{mmol})$ in $\mathrm{CH}_{2} \mathrm{Cl}_{2}(1.9$ $\mathrm{mL})$ at $0{ }^{\circ} \mathrm{C}$ were added tolualdehyde $(53 \mu \mathrm{L}, 0.45 \mathrm{mmol})$ and $\mathrm{InCl}_{3}$ (25 mg, $0.11 \mathrm{mmol}$ ). The solution was stirred at $0{ }^{\circ} \mathrm{C}$ for $2 \mathrm{~h}$, warmed to $\mathrm{rt}$ and stirred for and additional $30 \mathrm{~min}$. The mixture was quenched with saturated aqueous $\mathrm{NH}_{4} \mathrm{Cl}$ and extracted with $\mathrm{CH}_{2} \mathrm{Cl}_{2}$. The combined organic extracts were washed with $\mathrm{H}_{2} \mathrm{O}$, brine, dried $\left(\mathrm{Na}_{2} \mathrm{SO}_{4}\right)$, and concentrated. Purification by silica-gel chromatography $\left(\mathrm{CH}_{2} \mathrm{Cl}_{2}\right.$-benzene, $\left.1: 4\right)$ gave a colorless oil (44 mg, 74\%); ${ }^{1} \mathrm{H}$ NMR (300 MHz, $\mathrm{CDCl}_{3}$ ) $\delta 1.04$ (d, $\left.J=7.2 \mathrm{~Hz}, 3 \mathrm{H}\right), 2.38$ (s, $3 \mathrm{H}), 2.62(\mathrm{ddd}, J=1.2,3.0,14.6 \mathrm{~Hz}, 1 \mathrm{H}), 2.76(\mathrm{qdd}, J=1.1,2.6,7.2$ $\mathrm{Hz}, 1 \mathrm{H}), 2.87(\mathrm{dd}, J=11.8,14.6 \mathrm{~Hz}, 1 \mathrm{H}), 4.83(\mathrm{dd}, J=2.9,11.8 \mathrm{~Hz}$, $1 \mathrm{H}), 4.99(\mathrm{~d}, J=2.5 \mathrm{~Hz}, 1 \mathrm{H}), 7.20(\mathrm{~d}, J=8.0 \mathrm{~Hz}, 2 \mathrm{H}), 7.30(\mathrm{~d}, J=8.0$ $\mathrm{Hz}, 2 \mathrm{H}), 7.32-7.55(\mathrm{~m}, 5 \mathrm{H}) ;{ }^{13} \mathrm{C} \mathrm{NMR}\left(75 \mathrm{MHz}, \mathrm{CDCl}_{3}\right) \delta 11.3,21.1$, 45.7, 50.9, 78.8, 80.1, 125.3, 125.5, 127.9, 128.6, 128.9, 135.4, 136.9, 141.0, 210.7; IR (neat) 2976, 1714, $1099 \mathrm{~cm}^{-1}$; HRMS $\left(\mathrm{M}+\mathrm{H}^{+}\right)$ calcd for $\mathrm{C}_{19} \mathrm{H}_{21} \mathrm{O}_{2}$ 281.1536, found 281, 1538 .

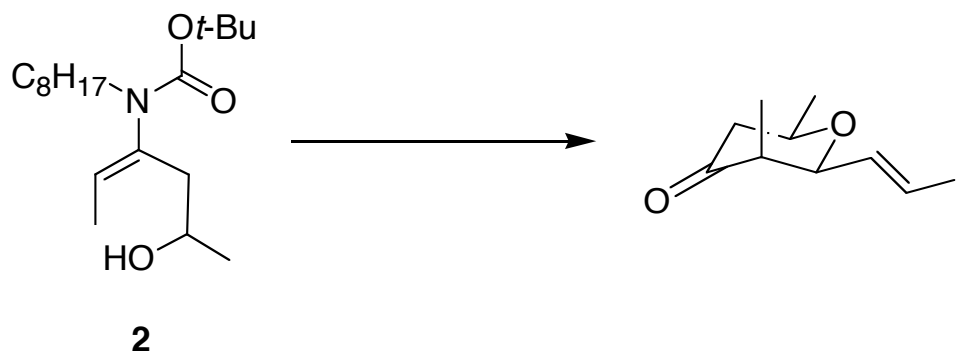

3,6-Dimethyl-2-propenyltetrahydropyran-4-one of entry 4 . To a solution of alcohol $2(150 \mathrm{mg}, 0.46 \mathrm{mmol})$ in $\mathrm{CH}_{2} \mathrm{Cl}_{2}(3.8 \mathrm{~mL})$ at $0{ }^{\circ} \mathrm{C}$ were added crotonaldehyde $(76 \mu \mathrm{L}, 0.92 \mathrm{mmol})$ and $\mathrm{InCl}_{3}(51$ $\mathrm{mg}, 0.23 \mathrm{mmol})$. The solution was stirred at $0{ }^{\circ} \mathrm{C}$ for $4 \mathrm{~h}$, and warmed to $\mathrm{rt}$ over $25 \mathrm{~min}$. The mixture was quenched with saturated aqueous $\mathrm{NH}_{4} \mathrm{Cl}$ and extracted with $\mathrm{CH}_{2} \mathrm{Cl}_{2}$. The combined organic extracts were washed with $\mathrm{H}_{2} \mathrm{O}$, brine, dried $\left(\mathrm{Na}_{2} \mathrm{SO}_{4}\right)$, and 
concentrated. Purification by silica-gel chromatography (ethyl acetate-hexanes, $1: 19)$ gave a colorless oil (70 mg, 91\%); ${ }^{1} \mathrm{H}$ NMR $\left(300 \mathrm{MHz}, \mathrm{CDCl}_{3}\right) \delta 1.09(\mathrm{~d}, J=7.2 \mathrm{~Hz}, 3 \mathrm{H}), 1.29(\mathrm{~d}, J=6.1 \mathrm{~Hz}, 3 \mathrm{H})$, $1.68(\mathrm{dd}, J=1.6,6.5 \mathrm{~Hz}, 3 \mathrm{H}), 2.17(\mathrm{ddd}, J=1.2,2.8,14.6 \mathrm{~Hz}, 1 \mathrm{H})$, 2.30 (qdd, $J=1.2,2.7,7.2 \mathrm{~Hz}, 1 \mathrm{H}), 2.37$ (dd, $J=11.5,14.6 \mathrm{~Hz}, 1 \mathrm{H})$, $3.72(\mathrm{dqd}, J=2.8,6.1,11.5 \mathrm{~Hz}, 1 \mathrm{H}), 4.09(\mathrm{~m}, 1 \mathrm{H}), 5.43(\mathrm{ddq}, J=1.6$, 6.3, $15.4 \mathrm{~Hz}, 1 \mathrm{H}), 5.72$ (dqd, $J=1.2,6.5,15.4 \mathrm{~Hz}, 1 \mathrm{H}) ;{ }^{13} \mathrm{C}$ NMR $(75$ $\left.\mathrm{MHz}, \mathrm{CDCl}_{3}\right) \delta 11.1,17.8,22.0,45.5,49.7,73.1,79.4,127.5,128.5$, 211.2; IR (neat) 2974, 1715, $1092 \mathrm{~cm}^{-1}$; HRMS $\left(\mathrm{M}+\mathrm{H}^{+}\right)$calcd for $\mathrm{C}_{10} \mathrm{H}_{17} \mathrm{O}_{2}$ 169.1223, found 169.1209.

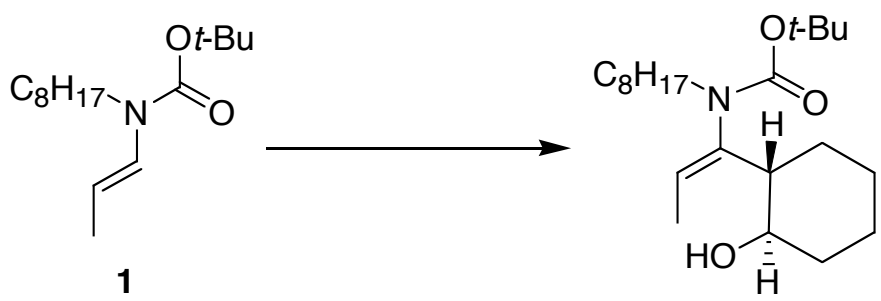

$t$ e $r t-B$ uty 1

$N$-octyl- $N$ - $[(E)-1-(2-h y d r o x y c y c l o h e x y l)-$ propenyl]carbamate. To a solution of enecarbamate 1 (350 $\mathrm{mg}$ $1.3 \mathrm{mmol})$ in THF $(1.2 \mathrm{~mL})$ at $-78^{\circ} \mathrm{C}$ was added dropwise over $5 \mathrm{~min}$ t-BuLi $(1.7 \mathrm{M}$ in pentane, $382 \mu \mathrm{L}, 0.65 \mathrm{mmol})$. The solution was stirred at $-78{ }^{\circ} \mathrm{C}$ for $30 \mathrm{~min}$, warmed to $-50{ }^{\circ} \mathrm{C}$ and stirred for an additional $30 \mathrm{~min}$. The mixture was cooled to $-78{ }^{\circ} \mathrm{C}$ and $\mathrm{BF}_{3} \cdot \mathrm{OEt}_{2}$ (148 $\mu \mathrm{L}, 1.2 \mathrm{mmol}$ ) was added, followed by cyclohexene oxide (66 $\mu \mathrm{L}, 0.65 \mathrm{mmol})$. The solution was stirred at $-78{ }^{\circ} \mathrm{C}$ for $5 \mathrm{~h}$, quenched with saturated aqueous $\mathrm{NH}_{4} \mathrm{Cl}$, and extracted with ethyl acetate. The combined organic extracts were washed with $\mathrm{H}_{2} \mathrm{O}$, brine, dried $\left(\mathrm{Na}_{2} \mathrm{SO}_{4}\right)$, and concentrated. Purification by silica-gel chromatography (ethyl acetate-hexanes, $1: 9$ ) gave a colorless oil (126 mg, 53\%); ${ }^{1} \mathrm{H}$ NMR $\left(300 \mathrm{MHz}, \mathrm{CDCl}_{3}\right.$ ) $\delta 0.85$ (br t, $J=6.6 \mathrm{~Hz}$, $3 \mathrm{H}), 1.23(\mathrm{~m}, 14 \mathrm{H}), 1.44(\mathrm{~s}, 9 \mathrm{H}), 1.49-1.77(\mathrm{~m}, 5 \mathrm{H}), 1.72(\mathrm{~d}, J=7.0$ $\mathrm{Hz}, 3 \mathrm{H}), 2.02(\mathrm{~m}, 1 \mathrm{H}), 2.47(\mathrm{~m}, 1 \mathrm{H}), 2.80(\mathrm{~m}, 1 \mathrm{H}), 3.24(\mathrm{~m}, 1 \mathrm{H}), 3.43$ $(\mathrm{m}, 1 \mathrm{H}), 4.91(\mathrm{br} \mathrm{s}, 1 \mathrm{H}), 5.54(\mathrm{q}, J=7.0 \mathrm{~Hz}, 1 \mathrm{H}) ;{ }^{13} \mathrm{C}$ NMR $(75 \mathrm{MHz}$, $\left.\mathrm{CDCl}_{3}\right) \delta 13.2,14.0,22.6,24.9,25.9,26.8,28.3,28.5,29.2,29.8$, $31.7,33.6,48.8,50.9,70.4,80.1,128.6,141.0,157.0$; IR (neat) 3444, 1679, $1164 \mathrm{~cm}^{-1}$; HRMS $\left(\mathrm{M}+\mathrm{H}^{+}\right)$calcd for $\mathrm{C}_{22} \mathrm{H}_{42} \mathrm{NO}_{3} 368.3159$, found 368.3161 . 

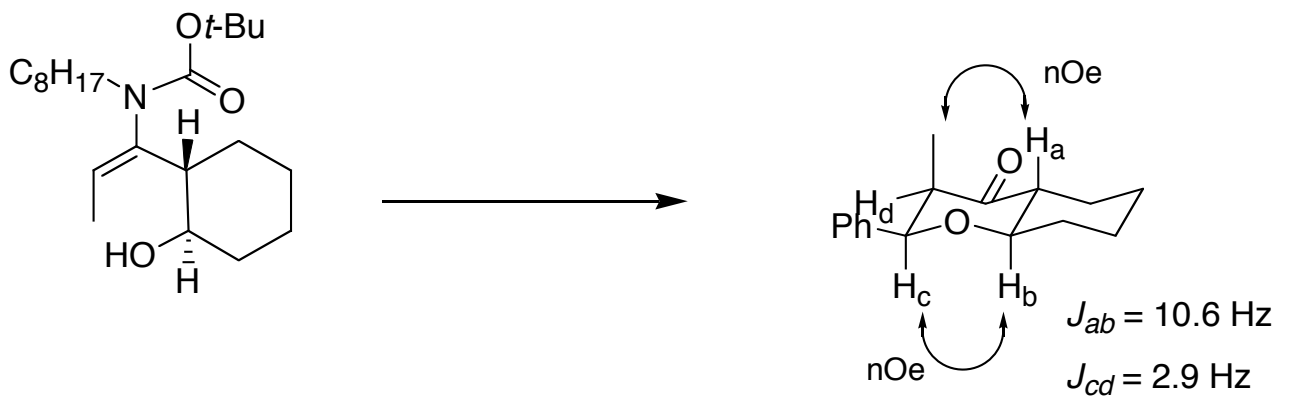

3-Methyl-2-phenyloctahydrochromen-4-one of entry 5. To a solution of tert-butyl $N$-octyl- $N-[(E)-1-(2$-hydroxycyclohexyl)propenyl]carbamate $(61 \mathrm{mg}, 0.17 \mathrm{mmol})$ in $\mathrm{CH}_{2} \mathrm{Cl}_{2}(1.39 \mathrm{~mL})$ were added benzaldehyde $(34 \mu \mathrm{L}, 0.33 \mathrm{mmol})$ and $\mathrm{InCl}_{3}(19 \mathrm{mg}, 0.08$ $\mathrm{mmol})$. The solution was stirred at $\mathrm{rt}$ for $90 \mathrm{~min}$, quenched with saturated aqueous $\mathrm{NH}_{4} \mathrm{Cl}$, and extracted with $\mathrm{CH}_{2} \mathrm{Cl}_{2}$. The combined organic extracts were washed with $\mathrm{H}_{2} \mathrm{O}$, brine, dried $\left(\mathrm{Na}_{2} \mathrm{SO}_{4}\right)$, and concentrated. Purification by silica-gel chromatography (benzene) gave a colorless oil (34 mg, 84\%); ${ }^{1} \mathrm{H}$ NMR (300 MHz, $\left.\mathrm{CDCl}_{3}\right) \delta 0.93$ $(\mathrm{d}, J=7.2 \mathrm{~Hz}, 3 \mathrm{H}), 1.28(\mathrm{~m}, 3 \mathrm{H}), 1.64(\mathrm{~m}, 1 \mathrm{H}), 1.83(\mathrm{~m}, 2 \mathrm{H}), 2.00(\mathrm{~m}$, $1 \mathrm{H}), 2.19(\mathrm{~m}, 1 \mathrm{H}), 2.47(\mathrm{td}, J=3.2,10.6 \mathrm{~Hz}, 1 \mathrm{H}), 2.69(\mathrm{qd}, J=2.9$, $7.2 \mathrm{~Hz}, 1 \mathrm{H}), 3.35(\mathrm{td}, J=3.9,10.6 \mathrm{~Hz}, 1 \mathrm{H}), 4.87(\mathrm{~d}, J=2.9 \mathrm{~Hz}, 1 \mathrm{H})$, 7.23-7.29 (m, 5H); ${ }^{13} \mathrm{C}$ NMR (75 MHz, $\left.\mathrm{CDCl}_{3}\right) \delta 11.9,23.1,24.4,24.8$, 33.1, 50.9, 51.5, 80.9, 81.7, 125.5, 127.2, 128.2, 138.8, 212.3; IR (neat) $2935,1711,1106 \mathrm{~cm}^{-1}$; HRMS $\left(\mathrm{M}+\mathrm{H}^{+}\right)$calcd for $\mathrm{C}_{16} \mathrm{H}_{21} \mathrm{O}_{2}$ 245.1536, found 245.1514 .
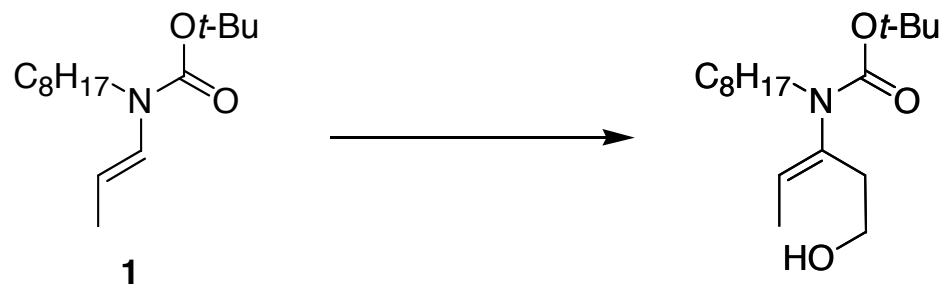

$t$ e $r t$-Butyl $\quad N$-octyl- $N-[(E)-1-(2-H y d r o x y e t h y l)$ propenyl $]-$ carbamate. To a solution of enecarbamate $1(1.00 \mathrm{~g}, 3.7 \mathrm{mmol})$ in THF $(37.1 \mathrm{~mL})$ at $-78^{\circ} \mathrm{C}$ was added dropwise over 4 min $s$-BuLi (1.4 $\mathrm{M}$ in cyclohexane, $3.71 \mathrm{~mL}, 5.2 \mathrm{mmol}$ ). The solution was stirred at $78{ }^{\circ} \mathrm{C}$ for $50 \mathrm{~min}$, warmed to $-30{ }^{\circ} \mathrm{C}$ and stirred for an additional 15 min. The mixture was cooled to $-78{ }^{\circ} \mathrm{C}$ and $\mathrm{BF}_{3} \cdot \mathrm{OEt}_{2}(1.41 \mathrm{~mL}, 11.1$ mmol) was added, followed by ethylene oxide $(1.85 \mathrm{~mL}, 37.1 \mathrm{mmol})$. The solution was stirred at $-78{ }^{\circ} \mathrm{C}$ for $6 \mathrm{~h}$. The mixture was 
quenched with saturated aqueous $\mathrm{NH}_{4} \mathrm{Cl}$ and extracted with ethyl acetate. The combined organic extracts were washed with $\mathrm{H}_{2} \mathrm{O}$, brine, dried $\left(\mathrm{Na}_{2} \mathrm{SO}_{4}\right)$, and concentrated. Purification by silica-gel chromatography (ethyl acetate-hexanes, $1: 9$ ) gave a colorless oil (616 mg, 53\%); ${ }^{1} \mathrm{H}$ NMR $\left(300 \mathrm{MHz}, \mathrm{CDCl}_{3}\right) \delta 0.85$ (br t, $J=6.7 \mathrm{~Hz}$, $3 \mathrm{H}), 1.24(\mathrm{~m}, 10 \mathrm{H}), 1.43(\mathrm{~s}, 9 \mathrm{H}), 1.47(\mathrm{~m}, 2 \mathrm{H}), 1.69(\mathrm{~d}, J=7.0 \mathrm{~Hz}$, $3 \mathrm{H}$ ), 2.34 (br t, $J=5.5 \mathrm{~Hz}, 2 \mathrm{H}$ ), 3.14 (br t, $J=7.5 \mathrm{~Hz}, 2 \mathrm{H}$ ), 3.58 (br q, $J=6.2 \mathrm{~Hz}, 2 \mathrm{H}$ ), 3.99 (v br s, $1 \mathrm{H}), 5.55$ (br q, $J=6.1 \mathrm{~Hz}, 1 \mathrm{H}) ;{ }^{13} \mathrm{C}$ NMR $\left(75 \mathrm{MHz}, \mathrm{CDCl}_{3}\right) \delta 13.1,14.0,22.6,26.7,28.3,28.4,29.2,31.7,31.9$, 47.6, 59.1, 80.1, 128.2, 135.9, 156.5; IR (neat) 3454, 1697, $1166 \mathrm{~cm}^{-}$ ${ }^{1}$; HRMS $\left(\mathrm{M}+\mathrm{H}^{+}\right)$calcd for $\mathrm{C}_{18} \mathrm{H}_{36} \mathrm{NO}_{3} 314.2690$, found 314.2678.

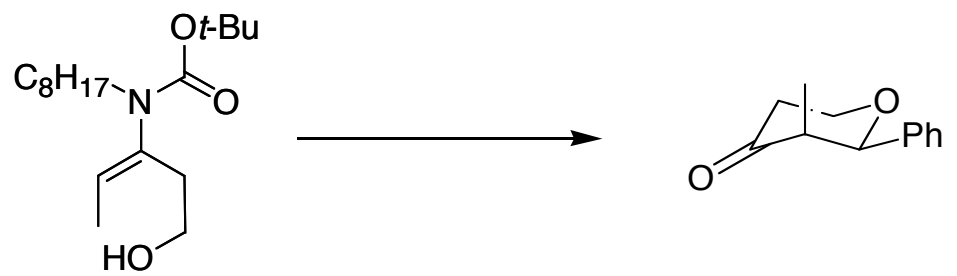

3-Methyl-2-phenyl-tetrahydropyran-4-one of entry 6. To a solution of tert-butyl $\mathrm{N}$-octyl- $\mathrm{N}-[(E)-1-(2-\mathrm{Hyd}$ roxyethyl)propenyl]carbamate $(144 \mathrm{mg}, 0.46 \mathrm{mmol})$ in $\mathrm{CH}_{2} \mathrm{Cl}_{2}(3.8 \mathrm{~mL})$ at $0{ }^{\circ} \mathrm{C}$ were added benzaldehyde $(93 \mu \mathrm{L}, 0.91 \mathrm{mmol})$ and $\mathrm{InCl}_{3}(51 \mathrm{mg}, 0.23$ $\mathrm{mmol})$. The solution was stirred at $0{ }^{\circ} \mathrm{C}$ for $1 \mathrm{~h}$, warmed to $\mathrm{rt}$ and stirred for an additional $3 \mathrm{~h}$. The mixture was quenched with saturated aqueous $\mathrm{NH}_{4} \mathrm{Cl}$ and extracted with $\mathrm{CH}_{2} \mathrm{Cl}_{2}$. The combined organic extracts were washed with $\mathrm{H}_{2} \mathrm{O}$, brine, dried $\left(\mathrm{Na}_{2} \mathrm{SO}_{4}\right)$, and concentrated. Purification by silica-gel chromatography (ethyl acetate-hexanes, 1 : 9) gave a colorless oil (72 mg, 83\%); ${ }^{1} \mathrm{H}$ NMR $\left(300 \mathrm{MHz}, \mathrm{CDCl}_{3}\right) \delta 0.92(\mathrm{~d}, J=7.2 \mathrm{~Hz}, 3 \mathrm{H}), 2.30(\mathrm{dp}, J=1.6,14.8$ $\mathrm{Hz}, 1 \mathrm{H}$ ), 2.70 (qdd, $J=1.4,3.0,7.2 \mathrm{~Hz}, 1 \mathrm{H}$ ), 2.88 (ddd, $J=7.6,12.0$, $14.8 \mathrm{~Hz}, 1 \mathrm{H}), 3.81$ (ddd, $J=3.3,11.5,11.9 \mathrm{~Hz}, 1 \mathrm{H}), 4.39$ (ddd, $J=$ 1.7, 7.6, $11.4 \mathrm{~Hz}, 1 \mathrm{H}), 4.81(\mathrm{~d}, J=3.0 \mathrm{~Hz}, 1 \mathrm{H}), 7.24-7.39(\mathrm{~m}, 5 \mathrm{H}) ;{ }^{13} \mathrm{C}$ NMR $\left(75 \mathrm{MHz}, \mathrm{CDCl}_{3}\right) \delta 11.1,38.4,51.5,66.5,81.2,125.5,127.4$, 128.2, 138.4, 210.8; IR (neat) 1716, $1104 \mathrm{~cm}^{-1}$; LRMS (M+) 190. 
<smiles>CCC=O</smiles>

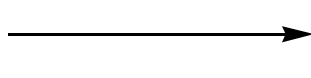<smiles>C=CN([PH2+]c1ccccc1)C(=O)O[Mg]C(C)(C)C</smiles>

tert-Butyl $N$-octyl- $N$-vinylcarbamate. To a mixture of octylamine (451 mg, $3.49 \mathrm{mmol})$ and $4 \AA$ mol sieves $(553 \mathrm{mg})$ in $\mathrm{CH}_{2} \mathrm{Cl}_{2}(4.4 \mathrm{~mL})$ at $0{ }^{\circ} \mathrm{C}$ was added acetaldehyde $(235 \mu \mathrm{L}, 4.20 \mathrm{mmol})$ dropwise. The solution was warmed to rt over $1 \mathrm{~h}$, stirred for an additional $1 \mathrm{~h}$, and decanted. To the resultant solution at $0{ }^{\circ} \mathrm{C}$ were added $\mathrm{CH}_{2} \mathrm{Cl}_{2}(4.4 \mathrm{~mL})$, di-tert-butyl dicarbonate (761 mg, 3.49 $\mathrm{mmol})$, and triethylamine $(729 \mu \mathrm{L}, 5.23 \mathrm{mmol})$. The mixture was warmed to $\mathrm{rt}$ and stirred for $18 \mathrm{~h}$. The solution was concentrated, and toluene $(4.4 \mathrm{~mL})$ was added. The solution was heated at $70{ }^{\circ} \mathrm{C}$ for $6 \mathrm{~h}$ and then concentrated. Purification by silica-gel chromatography (ethyl acetate-hexanes, 1 : 99) gave a colorless oil (489 mg, 55\%); ${ }^{1} \mathrm{H}$ NMR (300 MHz, $\mathrm{CDCl}_{3}$ ) $\delta 0.84$ (br t, $J=6.6 \mathrm{~Hz}$, $3 \mathrm{H}), 1.24(\mathrm{~m}, 1 \mathrm{OH}), 1.47(\mathrm{~s}, 9 \mathrm{H}), 1.50(\mathrm{~m}, 2 \mathrm{H}), 3.42$ (br s, 2H), 4.14 (br s, $1 \mathrm{H}), 4.22(\mathrm{~d}, J=16.1 \mathrm{~Hz}, 1 \mathrm{H}), 7.01$ (v br s, $1 \mathrm{H}) ;{ }^{13} \mathrm{C}$ NMR $(75$

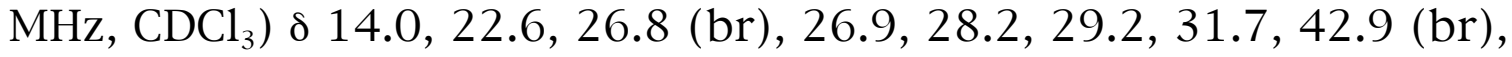
80.7, 90.2, 132.8, 152.9 (br); IR (neat) 2929, 1711, $1149 \mathrm{~cm}^{-1}$; HRMS $\left(\mathrm{M}+\mathrm{H}^{+}\right)$calcd for $\mathrm{C}_{15} \mathrm{H}_{30} \mathrm{NO}_{2} 256.2271$, found 256.2265.
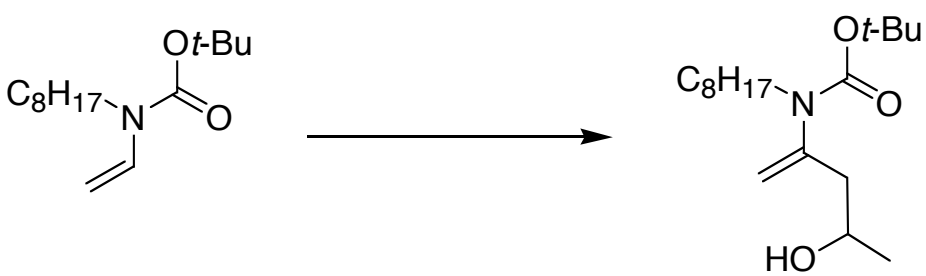

t e r t-Butyl $\quad N$-octyl- $N$-[1-(2-hydroxypropyl)vinyl]carbamate. To a solution of tert-butyl $N$-octyl- $N$-vinylcarbamate $(500 \mathrm{mg}, 1.96$ $\mathrm{mmol})$ in THF $(6.53 \mathrm{~mL})$ at $-78^{\circ} \mathrm{C}$ was added dropwise over $8 \mathrm{~min} \mathrm{t}$ BuLi $(1.7 \mathrm{M}$ in pentane, $1.09 \mathrm{~mL}, 1.86 \mathrm{mmol})$. The solution was stirred at $-78{ }^{\circ} \mathrm{C}$ for $50 \mathrm{~min}$, warmed to $-50{ }^{\circ} \mathrm{C}$ and stirred for an additional $25 \mathrm{~min}$. The mixture was cooled to $-78{ }^{\circ} \mathrm{C}$ and $\mathrm{BF}_{3} \cdot \mathrm{OEt}_{2}$ $(124 \mu \mathrm{L}, 0.98 \mathrm{mmol})$ was added, followed by propylene oxide $(69 \mu \mathrm{L}$, $0.98 \mathrm{mmol})$. The solution was slowly warmed to $-20{ }^{\circ} \mathrm{C}$ over $2.5 \mathrm{~h}$. The mixture was quenched with saturated aqueous $\mathrm{NH}_{4} \mathrm{Cl}$ and extracted with ethyl acetate. The combined organic extracts were washed with $\mathrm{H}_{2} \mathrm{O}$, brine, dried $\left(\mathrm{Na}_{2} \mathrm{SO}_{4}\right)$, and concentrated. 
Purification by silica-gel chromatography $\left(\mathrm{CH}_{2} \mathrm{Cl}_{2}\right.$, followed by $\mathrm{Et}_{2} \mathrm{O}$ $\left.\mathrm{CH}_{2} \mathrm{Cl}_{2}, 1: 19\right)$ gave a colorless oil (225 mg, 73\%); ${ }^{1} \mathrm{H}$ NMR $(300 \mathrm{MHz}$, $\left.\mathrm{CDCl}_{3}\right) \delta 0.87(\mathrm{br} \mathrm{t}, J=6.6 \mathrm{~Hz}, 3 \mathrm{H}), 1.20(\mathrm{~d}, J=6.2 \mathrm{~Hz}, 3 \mathrm{H}), 1.26(\mathrm{~m}$, $10 \mathrm{H}), 1.45(\mathrm{~s}, 9 \mathrm{H}), 1.51(\mathrm{~m}, 2 \mathrm{H}), 2.12(\mathrm{dd}, J=10.0,14.1 \mathrm{~Hz}, 1 \mathrm{H})$, 2.33 (br d, $J=14.0 \mathrm{~Hz}, 1 \mathrm{H}$ ), 3.04 (dt, $J=7.2,14.0 \mathrm{~Hz}, 1 \mathrm{H}$ ), 3.41 (dt, $J=7.8,14.0 \mathrm{~Hz}, 2 \mathrm{H}) 3.81(\mathrm{~m}, 1 \mathrm{H}) 4.97(\mathrm{~s}, 1 \mathrm{H}), 5.12(\mathrm{~s}, 1 \mathrm{H}) ;{ }^{13} \mathrm{C}$ NMR $\left(75 \mathrm{MHz}, \mathrm{CDCl}_{3}\right) \delta 14.0,22.2,22.6,26.6,28.3$, 28.4, 29.2, 31.7, 44.6, 47.3, 64.3, 80.5, 116.4, 144.3, 155.1; IR (neat) 3454, 1699, $1168 \mathrm{~cm}^{-}$ ${ }^{1}$; HRMS $\left(\mathrm{M}+\mathrm{H}^{+}\right)$calcd for $\mathrm{C}_{18} \mathrm{H}_{36} \mathrm{NO}_{3}$ 314.2690, found 314.2682.

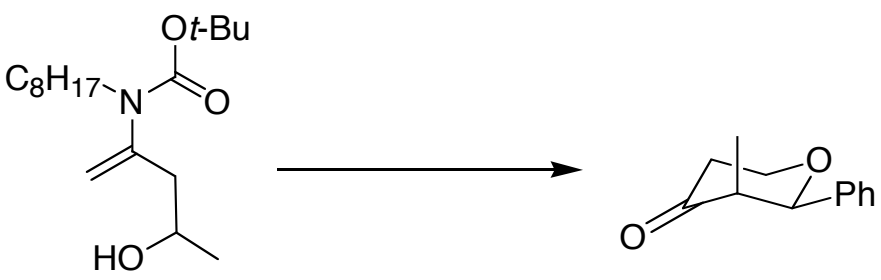

2-Methyl-6-phenyltetrahydropyran-4-one of entry 7. To a solution of tert-butyl $N$-octyl- $N$-[1-(2-hydroxypropyl)vinyl]carbamate $(80 \mathrm{mg}, 0.24 \mathrm{mmol})$ in pyridine $(0.81 \mathrm{~mL})$ at $0{ }^{\circ} \mathrm{C}$ was added TMSCl $(62 \mu \mathrm{L}, 0.48 \mathrm{mmol})$. The solution was warmed to $\mathrm{rt}$ and stirred for $21 \mathrm{~h}$, diluted with $\mathrm{CH}_{2} \mathrm{Cl}_{2}$, and quenched with $\mathrm{NaHCO}_{3}$. The aqueous layer was extracted with $\mathrm{CH}_{2} \mathrm{Cl}_{2}$, and combined organic extracts were washed with $\mathrm{H}_{2} \mathrm{O}$, brine, and dried $\left(\mathrm{Na}_{2} \mathrm{SO}_{4}\right)$. The solution was concentrated to provide the trimethylsilyl ether as a yellow oil (90 mg, 92\%) which was used without further purification; ${ }^{1} \mathrm{H}$ NMR $\left(200 \mathrm{MHz} \mathrm{CDCl}_{3}\right) \delta 0.10$ (s, 9H), 0.86 (br t, $J=7.0 \mathrm{~Hz}, 3 \mathrm{H}), 1.17-1.41(\mathrm{~m}, 14 \mathrm{H}), 1.35-1.52(\mathrm{~m}$, $10 \mathrm{H}), 1.66(\mathrm{~d}, J=7.0 \mathrm{~Hz}, 3 \mathrm{H}), 2.26(\mathrm{~m}, 1 \mathrm{H}), 2.41(\mathrm{dd}, J=9.0,13.8$ $\mathrm{Hz}, 1 \mathrm{H}), 3.08(\mathrm{dt}, 6.7,14.4 \mathrm{~Hz}, 1 \mathrm{H}), 3.35(\mathrm{~m}, 1 \mathrm{H}), 3.88(\mathrm{~m}, 1 \mathrm{H}), 5.40$ (br q, $J=6.9 \mathrm{~Hz}, 1 \mathrm{H}$ ).

To a solution of the trimethylsilyl ether $(48 \mathrm{mg}, 0.12 \mathrm{mmol})$ in acetonitrile $(1.24 \mathrm{~mL})$ at $-45{ }^{\circ} \mathrm{C}$ were added benzaldehyde $(15 \mu \mathrm{L}$, $0.15 \mathrm{mmol})$ and TMSOTf $(25 \mu \mathrm{L}, 0.14 \mathrm{mmol})$. The solution was stirred at $-45{ }^{\circ} \mathrm{C}$ for $40 \mathrm{~min}$, quenched with $\mathrm{NaHCO}_{3}$, and extracted with $\mathrm{CH}_{2} \mathrm{Cl}_{2}$. The combined organic extracts were washed with $\mathrm{H}_{2} \mathrm{O}$, brine, dried $\left(\mathrm{Na}_{2} \mathrm{SO}_{4}\right)$, and concentrated. Purification by silica-gel chromatography $\left(\mathrm{Et}_{2} \mathrm{O}\right.$-hexanes, $\left.1: 9\right)$ gave a colorless oil $(15 \mathrm{mg}$, 65\%); ${ }^{1} \mathrm{H}$ NMR (300 MHz, $\left.\mathrm{CDCl}_{3}\right) \delta 1.42(\mathrm{~d}, J=6.1 \mathrm{~Hz}, 3 \mathrm{H}), 2.37$ (dd, 
$J=11.0,14.3 \mathrm{~Hz}, 1 \mathrm{H}), 2.48(\mathrm{ddd}, J=1.6,2.9,14.3 \mathrm{~Hz}, 1 \mathrm{H}), 2.54-$ $2.65(\mathrm{~m}, 2 \mathrm{H}), 3.93(\mathrm{dqd}, J=2.9,6.1,11.0 \mathrm{~Hz}, 1 \mathrm{H}), 4.65(\mathrm{dd}, J=4.1$, $10.2 \mathrm{~Hz}, 1 \mathrm{H}), 7.25-7.41(\mathrm{~m}, 5 \mathrm{H}) ;{ }^{13} \mathrm{C} \mathrm{NMR}\left(75 \mathrm{MHz}, \mathrm{CDCl}_{3}\right) \delta 22.2$, 49.3, 73.6, 78.7, 125.7, 128.1, 128.7, 140.8, 206.9; IR (neat) 1720, $1057 \mathrm{~cm}^{-1}$; LRMS $\left(\mathrm{M}+\mathrm{H}^{+}\right)$191.2.
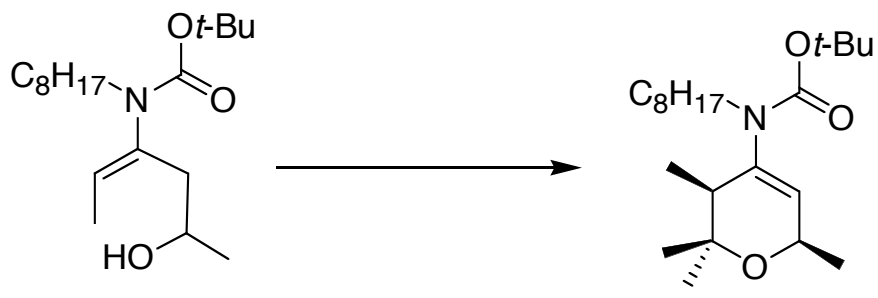

t e r t-Butyl $\quad \mathrm{N}$-octyl- $\mathrm{N}$-(2,2,3,6-tetramethyl-3,6-dihydro- $2 \mathrm{H}$ pyran-4-yl)carbamate of entry 8 . To a solution of alcohol 2 $(60 \mathrm{mg}, 0.18 \mathrm{mmol})$ in $\mathrm{CH}_{2} \mathrm{Cl}_{2}(1.5 \mathrm{~mL})$ were added acetone $(135 \mu \mathrm{L}$, $1.8 \mathrm{mmol})$ and $\mathrm{InCl}_{3}(20 \mathrm{mg}, 0.09 \mathrm{mmol})$. The solution was stirred at $\mathrm{rt}$ for $24 \mathrm{~h}$, quenched with saturated aqueous $\mathrm{NH}_{4} \mathrm{Cl}$, and extracted with $\mathrm{CH}_{2} \mathrm{Cl}_{2}$. The combined organic extracts were washed with $\mathrm{H}_{2} \mathrm{O}$, brine, dried $\left(\mathrm{Na}_{2} \mathrm{SO}_{4}\right)$, and concentrated. Purification by silica-gel chromatography (ethyl acetate-hexanes, 1 : 19) gave a colorless oil (33 mg, 49\%); ${ }^{1} \mathrm{H}$ NMR $\left(300 \mathrm{MHz} \mathrm{CDCl}_{3}\right) \delta 0.86$ (br t, $J=$ $6.7 \mathrm{~Hz}, 3 \mathrm{H}), 1.01(\mathrm{~d}, J=6.9 \mathrm{~Hz}, 3 \mathrm{H}), 1.19(\mathrm{~s}, 3 \mathrm{H}), 1.23(\mathrm{~d}, J=6.6 \mathrm{~Hz}$, $3 \mathrm{H}), 1.26(\mathrm{~m}, 10 \mathrm{H}), 1.29(\mathrm{~s}, 3 \mathrm{H}), 1.43(\mathrm{~s}, 9 \mathrm{H}), 1.54(\mathrm{~m}, 2 \mathrm{H}), 2.00(\mathrm{br}$ q, $J=6.0 \mathrm{~Hz}, 1 \mathrm{H}), 3.27(\mathrm{~m}, 2 \mathrm{H}), 4.26(\mathrm{qt}, J=1.9,6.6 \mathrm{~Hz}, 1 \mathrm{H}), 5.34$ $(\mathrm{d}, J=1.6 \mathrm{~Hz}, 1 \mathrm{H}) ;{ }^{13} \mathrm{C}$ NMR $\left(75 \mathrm{MHz}, \mathrm{CDCl}_{3}\right) \delta 14.1,14.8,21.4,22.6$, 24.5, 26.8, 27.0, 28.4, 29.0, 29.2, 29.3, 31.8, 39.6, 48.7, 65.6, 73.6, 79.6, 125.0, 140.7, 154.7; IR (neat) 2974, 1688, $1145 \mathrm{~cm}^{-1}$; HRMS $\left(\mathrm{M}+\mathrm{H}^{+}\right)$calcd for $\mathrm{C}_{22} \mathrm{H}_{42} \mathrm{NO}_{3} 368.3159$, found 368.3163 . 


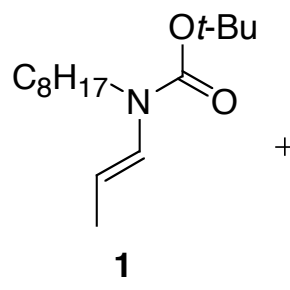

$(3 S)-t$ e $r t-\mathrm{Buty} 1$
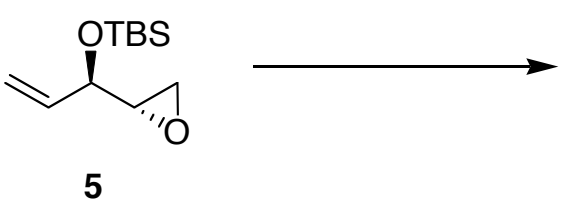

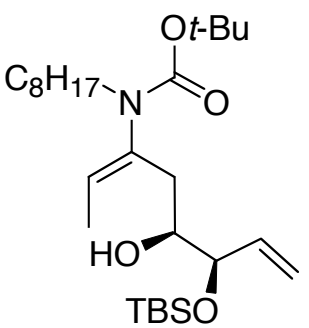

6 silanyloxy)-1-ethylidene-3-hydroxy-(E)-hex-5-enyl)-

carbamate (6). To a solution of enecarbamate 1 (251 mg, 0.93 $\mathrm{mmol})$ in THF $(0.93 \mathrm{~mL})$ at $-78{ }^{\circ} \mathrm{C}$ was added dropwise over $5 \mathrm{~min} \mathrm{t}^{-}$ BuLi $(1.7 \mathrm{M}$ in pentane, $494 \mu \mathrm{L}, 0.84 \mathrm{mmol})$. The solution was stirred at $-78{ }^{\circ} \mathrm{C}$ for $30 \mathrm{~min}$, warmed to $-50{ }^{\circ} \mathrm{C}$ and stirred for an additional $30 \mathrm{~min}$. The mixture was cooled to $-78{ }^{\circ} \mathrm{C}$ and $\mathrm{BF}_{3} \cdot \mathrm{OEt}_{2}$ $(106 \mu \mathrm{L}, 0.84 \mathrm{mmol})$ was added, followed by epoxide $5^{1}$ in THF $(0.15$ $\mathrm{mL})$. The solution was stirred at $-78^{\circ} \mathrm{C}$ for $3 \mathrm{~h}$. The mixture was quenched with saturated aqueous $\mathrm{NH}_{4} \mathrm{Cl}$ and extracted with ethyl acetate. The combined organic extracts were washed with $\mathrm{H}_{2} \mathrm{O}$, brine, dried $\left(\mathrm{Na}_{2} \mathrm{SO}_{4}\right)$, and concentrated. Purification by silica-gel chromatography $\left(\mathrm{CH}_{2} \mathrm{Cl}_{2}\right.$-hexanes, $1: 1$, followed by $\left.\mathrm{CH}_{2} \mathrm{Cl}_{2}\right)$ gave a colorless oil (140 mg, 62\%); $[\alpha]^{20}{ }_{\mathrm{D}}=+32.3\left(\mathrm{c} 0.99, \mathrm{CHCl}_{3}\right) ;{ }^{1} \mathrm{H} \mathrm{NMR}$ $\left(300 \mathrm{MHz}, \mathrm{CDCl}_{3}\right) \delta 0.04(\mathrm{~s}, 3 \mathrm{H}), 0.08(\mathrm{~s}, 3 \mathrm{H}), 0.86(\mathrm{t}, J=6.9 \mathrm{~Hz}, 3 \mathrm{H})$, $0.89(\mathrm{~s}, 9 \mathrm{H}), 1.25(\mathrm{~m}, 10 \mathrm{H}), 1.43(\mathrm{~s}, 9 \mathrm{H}), 1.48(\mathrm{~m}, 2 \mathrm{H}), 1.68(\mathrm{~d}, J=$ $7.0 \mathrm{~Hz}, 3 \mathrm{H}), 2.33$ (m, 2H), 3.04 (br dt, $J=7.2,14.1 \mathrm{~Hz}, 1 \mathrm{H}), 3.29$ (m, $1 \mathrm{H}), 3.52(\mathrm{~m}, 1 \mathrm{H}), 4.10$ (br t, $J=5.6 \mathrm{~Hz}, 1 \mathrm{H}), 4.25$ (v br s, $1 \mathrm{H}), 5.14$ $(\mathrm{dt}, J=1.3,10.2 \mathrm{~Hz}, 1 \mathrm{H}), 5.27(\mathrm{dt}, J=1.6,17.2 \mathrm{~Hz}, 1 \mathrm{H}), 5.52(\mathrm{q}, J=$ $6.7 \mathrm{~Hz}, 1 \mathrm{H}), 5.88$ (ddd, $J=5.9,10.4,17.2 \mathrm{~Hz}, 1 \mathrm{H}) ;{ }^{13} \mathrm{C}$ NMR $(75 \mathrm{MHz}$, $\left.\mathrm{CDCl}_{3}\right) \delta-4.8,-4.4,13.2,14.0,18.2,22.6,25.8,26.7,28.3,28.4,29.2$, 31.7, 32.3, 47.9, 72.1, 76.6, 80.0, 115.6, 127.4, 136.5, 138.9, 156.2; IR (neat) $3426,1664,1163 \mathrm{~cm}^{-1}$; HRMS $\left(\mathrm{M}+\mathrm{H}^{+}\right)$calcd for $\mathrm{C}_{27} \mathrm{H}_{54} \mathrm{NO}_{4} \mathrm{Si}$ 484.3817, found 484.3805 . 

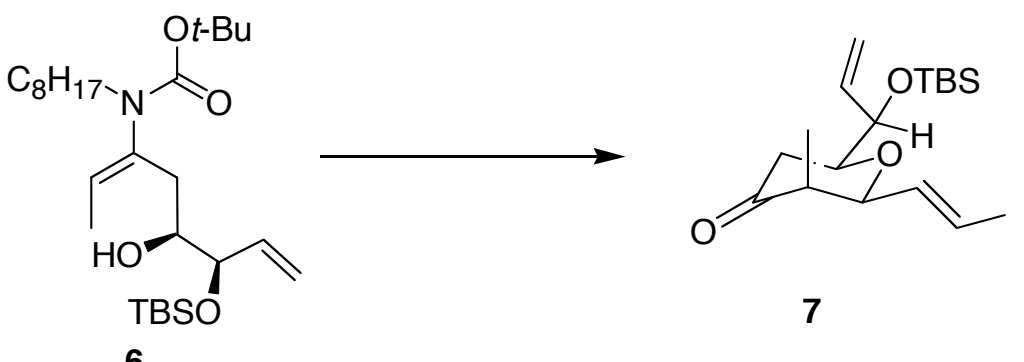

(2R,3R,6S)-6-[(1R)-1-(tert-Butyldimethylsilanyloxy)allyl]-3methyl-2-propenyltetrahydropyran-4-one (7). To a solution of alcohol $6(273 \mathrm{mg}, 0.56 \mathrm{mmol})$ in $\mathrm{CH}_{2} \mathrm{Cl}_{2}(4.7 \mathrm{~mL})$ at $0{ }^{\circ} \mathrm{C}$ were added crotonaldehyde $(93 \mu \mathrm{L}, 1.13 \mathrm{mmol})$ and $\mathrm{InCl}_{3}(67 \mathrm{mg}, 0.30$ mmol). The solution was stirred at $0{ }^{\circ} \mathrm{C}$ for $1 \mathrm{~h}$. The mixture was quenched with saturated aqueous $\mathrm{NH}_{4} \mathrm{Cl}$ and extracted with $\mathrm{CH}_{2} \mathrm{Cl}_{2}$. The combined organic extracts were washed with $\mathrm{H}_{2} \mathrm{O}$, brine, dried $\left(\mathrm{Na}_{2} \mathrm{SO}_{4}\right)$, and concentrated. Purification by silica-gel chromatography $\left(\mathrm{CH}_{2} \mathrm{Cl}_{2}\right.$-benzene, $\left.1: 19\right)$ gave a colorless oil (154 mg, 84\%); $[\alpha]^{20}{ }_{\mathrm{D}}=-14.8$ (c 1.0, $\left.\mathrm{CHCl}_{3}\right) ;{ }^{1} \mathrm{H}$ NMR $\left(300 \mathrm{MHz}, \mathrm{CDCl}_{3}\right) \delta$ 0.06 (s, 3H), 0.12 (s, 3H), 0.92 (s, 9H), 1.10 (d, J=7.2 Hz, 3H), 1.73 (br dt $J=1.4,6.5 \mathrm{~Hz}, 3 \mathrm{H}$ ), 2.13 (ddd, $J=1.4,2.9,14.9 \mathrm{~Hz}, 1 \mathrm{H}$ ), 2.33 (qdd, 1.4, 2.6, 7.2 Hz, 1H), 2.79 (dd, $J=11.6,14.9 \mathrm{~Hz}, 1 \mathrm{H}), 3.57$ (dt, $J=2.9,11.6 \mathrm{~Hz}, 1 \mathrm{H}), 4.12(\mathrm{~m}, 1 \mathrm{H}), 4.46(\mathrm{~m}, 1 \mathrm{H}), 5.14(\mathrm{dt}, 1.7,10.5$ $\mathrm{Hz}, 1 \mathrm{H}$ ), 5.29 (dt, $J=1.7,17.1 \mathrm{~Hz}, 1 \mathrm{H}$ ), 5.41 (ddq, $J=1.6,5.6,15.5$ $\mathrm{Hz}, 1 \mathrm{H}), 5.73(\mathrm{dqd}, J=1.4,6.5,15.4 \mathrm{~Hz}, 1 \mathrm{H}), 5.75(\mathrm{ddd}, J=5.0$, 10.5, $17.1 \mathrm{~Hz}, 1 \mathrm{H}) ;{ }^{13} \mathrm{C} \mathrm{NMR}\left(75 \mathrm{MHz}, \mathrm{CDCl}_{3}\right) \delta-4.2,-4.3,11.5,18.3$, 18.7, 26.2, 37.4, 50.6, 75.1, 79.7, 80.2, 116.4, 128.1, 128.2, 137.5, 213.0; IR (neat) 2929, 1716, $1096 \mathrm{~cm}^{-1}$; HRMS $\left(\mathrm{M}+\mathrm{H}^{+}\right)$calcd for $\mathrm{C}_{18} \mathrm{H}_{33} \mathrm{O}_{3} \mathrm{Si}$ 325.2194, found 325.2180.
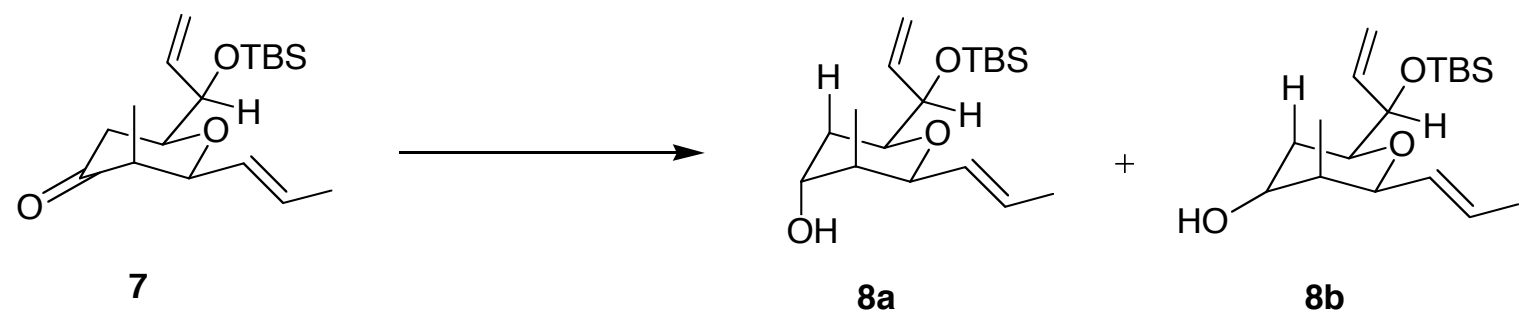

(2R,3R,6S)-6-[(1R)-1-(tert-Butyldimethylsilanyloxy)allyl]-3methyl-2-propenyltetrahydropyran-4-ol (8a) and $8 \mathrm{~b}$. To a solution of ketone $7(100 \mathrm{mg}, 0.31 \mathrm{mmol})$ in hexanes $(3.1 \mathrm{~mL})$ was added triisobutylaluminum (1.01 $\mathrm{M}$ in hexanes, $365 \mu \mathrm{L}, 0.36 \mathrm{mmol})$. 
The solution was stirred at $-30{ }^{\circ} \mathrm{C}$ for $90 \mathrm{~min}$, quenched with methanol, and stirred at $\mathrm{rt}$ with saturated aqueous potassium sodium tartrate for an additional $30 \mathrm{~min}$. The mixture was extracted with ether, and the combined organic extracts were washed with $\mathrm{H}_{2} \mathrm{O}$, brine, dried $\left(\mathrm{Na}_{2} \mathrm{SO}_{4}\right)$, and concentrated. Purification by silica-gel chromatography $\left(\mathrm{CH}_{2} \mathrm{Cl}_{2}\right)$ yielded a mixture (trans : cis, $48: 52$ ) as colorless oils (95 mg overall, 95\%); trans 8a: $[\alpha]_{\mathrm{D}}^{20}=-7.3\left(\mathrm{c} 1.6, \mathrm{CHCl}_{3}\right) ;{ }^{1} \mathrm{H} \mathrm{NMR}\left(300 \mathrm{MHz}, \mathrm{CDCl}_{3}\right) \delta 0.04(\mathrm{~s}, 3 \mathrm{H})$, 0.09 (s, 3H), 0.89 (d, J=7.2 Hz, 3H), 0.91 (s, 9H), $1.40(\mathrm{~m}, 1 \mathrm{H}), 1.56$ (br s, $1 \mathrm{H}), 1.59(\mathrm{~m}, 1 \mathrm{H}), 1.69(\mathrm{dt}, J=1.4,6.5 \mathrm{~Hz}, 3 \mathrm{H}), 1.88$ (ddd, $J=$ 2.8, 12.0, $14.4 \mathrm{~Hz}, 1 \mathrm{H}), 3.69$ (br dt, $J=3.0,12.0 \mathrm{~Hz}, 1 \mathrm{H}), 3.98(\mathrm{~m}$, $1 \mathrm{H}), 4.29(\mathrm{~m}, 1 \mathrm{H}), 4.38(\mathrm{~m}, 1 \mathrm{H}), 5.09(\mathrm{dt}, J=1.8,10.5 \mathrm{~Hz}, 1 \mathrm{H}), 5.26$ (dt, $J=1.8,17.2 \mathrm{~Hz}, 1 \mathrm{H}), 5.41$ (ddq, $J=1.6,5.6,15.4 \mathrm{~Hz}, 1 \mathrm{H}) 5.67$ $(\mathrm{dqd}, J=1.4,6.5,15.4 \mathrm{~Hz}, 1 \mathrm{H}), 5.80(\mathrm{ddd}, J=5.0,10.5,17.2 \mathrm{~Hz}$, $1 \mathrm{H}) ;{ }^{13} \mathrm{C}$ NMR $\left(75 \mathrm{MHz}, \mathrm{CDCl}_{3}\right) \delta-4.7,-4.6,11.1,17.9,18.3,25.9$, 26.9, 39.7, 70.5, 74.4, 75.4, 75.6, 114.9, 125.7, 130.6, 138.5; IR (neat) 3322, $1016 \mathrm{~cm}^{-1}$; HRMS $\left(\mathrm{M}+\mathrm{H}^{+}\right)$calcd for $\mathrm{C}_{18} \mathrm{H}_{35} \mathrm{O}_{3} \mathrm{Si} 327.2350$, found 327.2327; cis 8b: $[\alpha]^{20}{ }_{\mathrm{D}}=-15.2\left(c 0.55, \mathrm{CHCl}_{3}\right) ;{ }^{1} \mathrm{H}$ NMR $(300$ $\left.\mathrm{MHz}, \mathrm{CDCl}_{3}\right) \delta 0.03(\mathrm{~s}, 3 \mathrm{H}), 0.09(\mathrm{~s}, 3 \mathrm{H}), 0.83(\mathrm{~d}, J=6.9 \mathrm{~Hz}, 3 \mathrm{H})$, 0.90 (s, 9H), 1.60 (m, 2H), 1.70 (br dt, $J=1.4,6.5 \mathrm{~Hz}, 3 \mathrm{H}), 1.85(\mathrm{~m}$, $2 \mathrm{H}), 3.26(\mathrm{dt}, J=4.0,9.7 \mathrm{~Hz}, 1 \mathrm{H}), 3.80-3.93(\mathrm{~m}, 2 \mathrm{H}), 4.28(\mathrm{~m}, 1 \mathrm{H})$, 5.10 (dt, $J=1.7,10.5 \mathrm{~Hz}, 1 \mathrm{H}), 5.27$ (dt, $J=1.7,17.2 \mathrm{~Hz}, 1 \mathrm{H}), 5.43$ (ddq, $J=1.6,5.4,15.5 \mathrm{~Hz}, 1 \mathrm{H}), 5.66$ (dqd, $J=1.4,6.5,15.5 \mathrm{~Hz}, 1 \mathrm{H}$ ), $5.81(\mathrm{ddd}, J=5.2,10.5,17.2 \mathrm{~Hz}, 1 \mathrm{H}) ;{ }^{13} \mathrm{C} \mathrm{NMR}\left(75 \mathrm{MHz}, \mathrm{CDCl}_{3}\right) \delta$ 4.7, 5.1, 17.9, 18.2, 25.8, 28.2, 39.5, 71.2, 75.2, 79.1, 79.2, 115.1, 126.0, 130.0, 138.4; IR (neat) 3344, $1086 \mathrm{~cm}^{-1}$; HRMS $\left(\mathrm{M}+\mathrm{H}^{+}\right)$calcd for $\mathrm{C}_{18} \mathrm{H}_{35} \mathrm{O}_{3} \mathrm{Si} 327.2350$, found 327.2330.

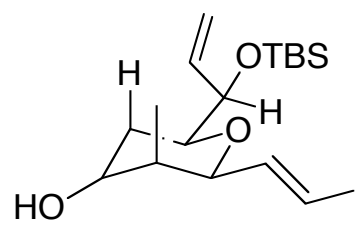

$8 b$

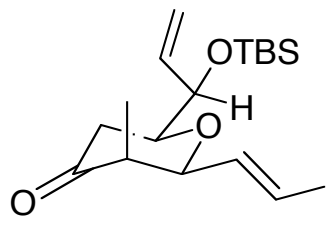

7

Oxidation of $8 \mathrm{~b}$. To a mixture of alcohol $8 \mathrm{~b}(135 \mathrm{mg}, 0.41 \mathrm{mmol})$ and $4 \AA$ mol sieves $(68 \mathrm{mg})$ in $\mathrm{CH}_{2} \mathrm{Cl}_{2}(4.2 \mathrm{~mL})$ at $0{ }^{\circ} \mathrm{C}$ were added $4-$ methylmorpholine- $N$-oxide $(73 \mathrm{mg}, 0.62 \mathrm{mmol})$ and tetrapropylammonium perruthenate $(12 \mathrm{mg}, 0.03 \mathrm{mmol})$. The mixture was stirred at $0{ }^{\circ} \mathrm{C}$ for $4 \mathrm{~h}$, filtered through a plug of silica gel, and 
concentrated. Purification by silica-gel chromatography $\left(\mathrm{Et}_{2} \mathrm{O}-\right.$ hexanes, 1 : 19) gave a colorless oil (132 mg, 98\%) identical to 7 , shown above.

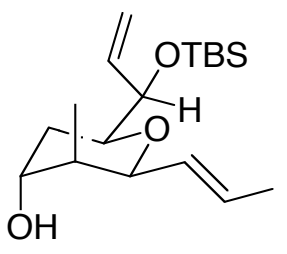

$8 a$

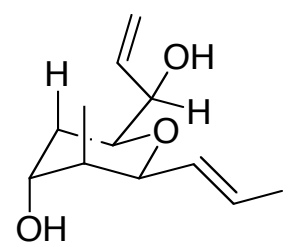

9

\section{6-(1-Hydroxyallyl)-3-methyl-2-propenyltetrahydropyran-4-}

ol (9). To a solution of alcohol $8 \mathrm{a}(43 \mathrm{mg}, 0.13 \mathrm{mmol})$ in THF $(2.20 \mathrm{~mL})$ at $\mathrm{rt}$ was added TBAF (1M in THF, $198 \mu \mathrm{L}, 0.20 \mathrm{mmol})$. The solution was stirred at $\mathrm{rt}$ for $20 \mathrm{~h}$, then additional TBAF (1M in THF, $198 \mu \mathrm{L}, 0.20 \mathrm{mmol}$ ) was added. The mixture was stirred at $\mathrm{rt}$ for $6 \mathrm{~h}$, quenched with saturated aqueous $\mathrm{NH}_{4} \mathrm{Cl}$ and extracted with ethyl acetate. The combined organic extracts were washed with $\mathrm{H}_{2} \mathrm{O}$, brine, dried $\left(\mathrm{Na}_{2} \mathrm{SO}_{4}\right)$, and concentrated. Purification by silica-gel chromatography (ethyl acetate : hexanes, $2: 3$ ) gave a thick colorless oil (28 mg, 100\%); $[\alpha]^{20}{ }_{\mathrm{D}}=-8.9\left(c 0.52, \mathrm{CHCl}_{3}\right) ;{ }^{1} \mathrm{H}$ NMR $\left(300 \mathrm{MHz}, \mathrm{CDCl}_{3}\right) \delta 0.89(\mathrm{~d}, J=7.2 \mathrm{~Hz}, 3 \mathrm{H}), 1.36(\mathrm{~m}, 1 \mathrm{H}), 1.63(\mathrm{~m}$, $1 \mathrm{H}), 1.71(\mathrm{dt}, J=1.3,6.4 \mathrm{~Hz}, 3 \mathrm{H}), 1.74(\mathrm{br} \mathrm{s}, 1 \mathrm{H}), 1.85$ (ddd, $J=2.9$, 12.3, 14.3 Hz, 1H), 2.48 (m, 1H), 3.88 (dt, $J=2.9,12.3 \mathrm{~Hz}, 1 \mathrm{H}), 3.99$ (br q, $J=2.8 \mathrm{~Hz}, 1 \mathrm{H}), 4.27(\mathrm{~m}, 1 \mathrm{H}), 4.45(\mathrm{~m}, 1 \mathrm{H}), 5.19$ (dt, $J=1.6$, $10.6 \mathrm{~Hz}, 1 \mathrm{H}), 5.31(\mathrm{dt}, J=1.6,17.3 \mathrm{~Hz}, 1 \mathrm{H}), 5.46(\mathrm{ddq}, J=1.6,6.3$, $15.4 \mathrm{~Hz}, 1 \mathrm{H}), 5.70(\mathrm{dqd}, J=1.2,6.4,15.4 \mathrm{~Hz}, 1 \mathrm{H}), 5.82(\mathrm{ddd}, J=5.9$, 10.6, $17.3 \mathrm{~Hz}, 1 \mathrm{H}) ;{ }^{13} \mathrm{C}$ NMR $\left(75 \mathrm{MHz}, \mathrm{CDCl}_{3}\right) \delta 11.1,17.9,26.7,39.6$, 70.1, 74.5, 74.6, 74.7, 116.5, 126.9, 130.0, 135.9; IR (neat) 3395, $1057 \mathrm{~cm}^{-1}$; HRMS $\left(\mathrm{M}+\mathrm{H}^{+}\right)$calcd for $\mathrm{C}_{12} \mathrm{H}_{21} \mathrm{O}_{3} 213.1485$, found 213.1466 .

\footnotetext{
${ }^{1}$ Epoxide 5 was prepared by Sharpless epoxidation of commercially available 1,4pentadien-3-ol followed by protection of the alcohol. (a) Epoxidation : Romero, A.; Wong, C. H.; J. Org. Chem. 2000, 65, 8264-8268. (b) The product of asymmetric epoxidation displayed an optical rotation of $[\alpha]^{20}=-64\left(c 0.73, \mathrm{CHCl}_{3}\right)$ compared to the literature value of $[\alpha]^{25}=-53\left(c 0.73, \mathrm{CHCl}_{3}\right)$ reported by Romero, et. al. (c) Protection as the TBS ether: Nicolaou, K. C.; Rodriquez, R. M.; Mitchell, H. J.; van Delft, F.L. Angew. Chem. Int. Ed. 1998, 37, 1874-1876. and refs. therein.
} 
${ }^{2}$ While the ${ }^{1} \mathrm{H}$ and ${ }^{13} \mathrm{C}$ NMR spectra of our compound are identical to those reported by Kalesse (Bhatt, U.; Christmann, M; Quitschalle, M.; Claus, E.; Kalesse, M. J. Org. Chem. 2001, 66, 1885-1893), the optical rotation is the opposite (-8.9) to what Kalesse reported (+9.2). Moreover, we observed that compound 37 in Kalesse's paper had a rotation of +14.3 which is the enantiomer of compound 39 in Williams' synthesis of (-)-ratjadone (Williams, D. R.; Ihle, D. C.; Plummer, S.V. Org. Lett. 2001, 3, 1383-1386, see supporting information), and has a very similar rotation $(+15)$. A similar situation pertains to aldehyde 38 of Kalesse's paper $(+19.4)$ and the enantiomeric aldehyde 8 in Williams' paper $(+23)$. Thus, it seems likely that Kalesse reported the rotations of the enantiomers of the compounds leading up to his pivotal intermediate 40 considering the fact that Kalesse prepared both enantiomers of compound 40 as reported in his additional biological studies (Kalesse, M.; Christmann, M.; Bhatt, U.; et al. ChemBiochem 2001, 2, 709-714). 